This document is the accepted manuscript version of the following article:

Pan, D., Hufenus, R., Qin, Z., Chen, L., \& Gooneie, A. (2019). Tuning gradient

microstructures in immiscible polymer blends by viscosity ratio. Journal of Applied

Polymer Science, 136(44), 48165 (9 pp.). https://doi.org/10.1002/app.48165

\title{
Tuning Gradient Microstructures in Immiscible Polymer Blends by Viscosity Ratio
}

\author{
Dan Pan ${ }^{\mathrm{a}, \mathrm{b}}$, Rudolf Hufenus ${ }^{\mathrm{a}}$, Zongyi Qin ${ }^{\mathrm{b}}$, Long Chen ${ }^{\mathrm{b} *}$, Ali Gooneie ${ }^{\mathrm{a} *}$
}

${ }^{a}$ Laboratory of Advanced Fibers, Empa, Swiss Federal Laboratories for Materials Science and Technology, Lerchenfeldstrasse 5, CH-9014 St. Gallen, Switzerland

${ }^{\mathrm{b}}$ State Key Laboratory for Modification of Chemical Fibers and Polymer Materials, College of Materials Science and Engineering, Donghua University, Shanghai 201620, P.R.China

*Corresponding Authors: (A.G.) ali.gooneie@empa.ch; (L.C.) happyjack@dhu.edu.cn

\begin{abstract}
Bio-inspired gradient microstructures provide an attractive template for functional materials with tailored properties. In this study, filaments with gradient microstructures are developed by melt-spinning of immiscible polymer blends. The distribution of the gradient morphology is shown to be controlled by the viscosity ratio of polymers as well as the geometry of the capillary die. Distinct microstructure gradients with long thin fibrils near the surface region and short large droplets near the center region of the filament, as well as the inverse pattern are formed in systems with different viscosity ratios. The shear flow field in the capillary can elucidate the formation mechanisms of gradient morphologies during processing. The results demonstrate how the features of a gradient microstructure can be tailored by the design of capillary geometry and processing conditions. The viscosity ratio is then introduced as an adjusting tool to control the gradient morphology in a given processing setup. In consequence, this study provides novel design routes for achieving gradient morphologies in immiscible polymers.
\end{abstract}




\section{Introduction}

The design of new functional materials often requires a precise control of the structure at different scales ${ }^{[1-9]}$. In polymer blends, this task is quite challenging due to the complex relationships between their properties, microstructures, and processing conditions. Recently, bio-inspired gradient polymer materials have attracted a lot of attention due to their characteristic morphologies and properties ${ }^{[10,11]}$. Most natural plants, such as bamboo, have different layers forming hierarchical microstructures with specific properties ${ }^{[12]}$. Cellulose fibers (vascular bundle) in bamboo are distributed densely in the outer region and sparsely in the inner region, which is beneficial to prevent buckling and fracture of bamboo under stress ${ }^{[13]}$. The tensile strength of the bamboo culm increases parabolically with radial distance and reaches its maximum value in the outer region due to the gradient distribution of fibers. By applying similar ideas to synthetic immiscible polymers, desirable morphologies and properties can be achieved by controlling the external field (e.g. gradient temperature field and electric fields $)^{[10 \text {, }}$ ${ }^{14]}$, polymer diffusion in interpenetrating polymer networks ${ }^{[15]}$, and phase separation in multicomponent blends ${ }^{[16]}$. Recently, we produced polymer fibers with gradient microstructures by tuning melt processing parameters and capillary die design ${ }^{[16-19]}$. We showed that gradient fibers with fine thin fibrils in the surface region and large slightly-deformed droplets in the central region can be achieved by special capillary design ${ }^{[19]}$. At the surface of such fibers, submicron channels aligned along the fiber axis were obtained by solvent etching, resulting in significantly altered surface properties. The formation of gradient structures has been shown to be triggered either by temperature gradients ${ }^{[16,18]}$ or by shear field gradients ${ }^{[19]}$. As for the latter mechanism, we aim to show here how the direction of gradient morphologies can be smartly reversed by a proper material selection based on their viscosity ratio.

Several theoretical and experimental studies have been performed to investigate the effect of viscosity ratio $p=\eta_{d} / \eta_{m}$ (where $\eta_{d}$ and $\eta_{m}$ are viscosity of dispersed phase and matrix, 
respectively) on the morphology of polymer blends ${ }^{[20-23]}$. The dimensionless parameter, capillary number $\mathrm{Ca}=\eta_{\mathrm{m}} \dot{\gamma} \mathrm{R}_{0} / \alpha$ (where $\dot{\gamma}$ is the shear rate, $\mathrm{R}_{0}$ the original radius of droplet and $\alpha$ the interfacial tension of polymer blends), is often used to expresses the ability of a droplet to deform or break up under the hydrodynamic equilibrium between viscous forces and interfacial tension under flow ${ }^{[24]}$. $\operatorname{Cox}^{[25]}$ and Grace ${ }^{[26]}$ predicted the droplet size during simple melt processing and proposed the deformation of a droplet to be $\mathrm{D}=\left(\frac{\mathrm{Ca}}{2}\right)\left[\frac{(19 \mathrm{p}+16) /(16 \mathrm{p}+16)}{\left((19 \mathrm{p} C \mathrm{Ca} / 40)^{2}+1\right)^{1 / 2}}\right]$ for all viscosity ratios under shear flow. Theoretically, a high viscosity ratio will result in large deformed droplets and a coarse morphology, whereas a low or matching viscosity ratio could result in fine fibrils and uniform morphology ${ }^{[19]}$. In most experimental cases, the dispersed phase was found to be finer for viscosity ratios less than one $(\mathrm{p} \leq 1)$ than that for viscosity ratios larger than one $(p>1)^{[27-30]}$. In addition to the droplet deformation and breakup differences in low and high viscosity ratio polymer blends, the migration of components, which also depends on the viscosity ratio ${ }^{[31]}$, is of significance for controlling the polymer morphology ${ }^{[32,33]}$. In a study on cellulose/aromatic polysulfonamide (PSA) alloy fibers with core-sheath structures, the low viscosity PSA phase was found to migrate to the outer layer of a filament during spinning $^{[32]}$. This migration facilitated the concentration of flame-retardant PSA in the surface region and improved the flame-retardant properties of blend fibers. There are indeed many factors influencing the morphology developments in immiscible polymer blends ${ }^{[34]}$. In this context, the dynamic mechanisms of deformation, breakup, and coalescence, are expressed in terms of various material properties. They are shown, both experimentally and theoretically, to depend on the viscoelastic behavior of each component as well as the processing conditions. Despite these efforts, new processes are still defined and characterized largely based on the most fundamental and accessible parameters (such as the viscosity ratio) at least during the early stages of their development. Furthermore, the viscosity ratio has been shown to be a 
primary parameter governing the morphology of immiscible polymer blends by pioneering works $^{[20,35]}$ as well as in more recent research ${ }^{[27,29]}$.

The gradient structures in polymer blends originates mainly from the structural reformations of the processed material due to the gradient of the flow field in the capillary $\operatorname{die}^{[19,36,37]}$. The development of radial and axial flow fields in a capillary are complex and largely determined by capillary length, diameter and entrance angle ${ }^{[38,39]}$. However, once the capillary geometry is fixed, the possibilities to tune the gradient structure are limited due to the fixed flow fields. In this paper, we investigate the effect of capillary geometry to complement previous studies regarding the role of capillary diameter and the transition of flow from the conical entrance to the capillary. The viscosity ratio is then introduced as a switch to reverse the gradient morphology in a given capillary setup. The formation mechanism of gradient morphology is evaluated based on experimental results and theoretical predictions.

\section{Experimental details}

\subsection{Materials}

Two types of polypropylene (PP) with highly differing melt flow indices were selected to satisfy the variation of viscosity ratio: PP1: polypropylene (HSP165G) with melt flow index of $16.5 \mathrm{~g} / 10 \mathrm{~min}\left(230{ }^{\circ} \mathrm{C} / 2.16 \mathrm{~kg}\right)$ was obtained from RESINEX Switzerland AG; PP2: polypropylene (PCR02) with melt flow index of $1.7 \mathrm{~g} / 10 \mathrm{~min}\left(230{ }^{\circ} \mathrm{C} / 2.16 \mathrm{~kg}\right)$ was obtained from Sabic. Polystyrene (PS, STYRON 643) with melt flow index of $3.5 \mathrm{~g} / 10 \mathrm{~min}$ $\left(200{ }^{\circ} \mathrm{C} / 5 \mathrm{~kg}\right.$ ) was obtained from Trinseo Europe $\mathrm{GmbH}$ (Switzerland).

\subsection{Rheological measurements}

The rheological properties of the polymers were measured using a rotary rheometer (MCR 303, Anton Paar $\mathrm{GmbH}$ ) with a parallel plate fixture. After loading the samples into the cell, temperature was maintained at $230{ }^{\circ} \mathrm{C}$ for 4 min to melt the polymers, and then it was 
equilibrated for $30 \mathrm{~s}$ prior to the rheological measurements. The shear viscosity of each polymer was measured. Steady sweeps were performed with shear rates ranging from $10^{-2}$ to $10^{1} \mathrm{~s}^{-1}$. The strain amplitude sweeps were conducted at an angular frequency of $10 \mathrm{rad} / \mathrm{s}$. The linear viscoelastic region, indicating the deformation range in which the test can be carried out without destroying the structure of polymers, was determined to be below $\sim 10 \%$. The frequency sweeps of PP1, PP2, and PS were then carried out at a constant strain amplitude of $5 \%$. The storage moduli of the polymers were then utilized to estimate the elasticity ratios of PS/PP1 and PS/PP2 blends as $\frac{\mathrm{G}_{\mathrm{PS}}^{\prime}}{\mathrm{G}_{\mathrm{PP}}^{\prime}}$, see Figure S1. All rheological measurements were repeated three times to demonstrate reproducibility.

\subsection{Sample preparation}

PP and PS granules were physically mixed with a constant blend ratio of $80 / 20 \mathrm{wt} / \mathrm{wt} \%$ in a mechanical mixer, and subsequently dried at $70{ }^{\circ} \mathrm{C}$ overnight under vacuum to minimize moisture content. The polymer mixture was melt-spun on a lab-scale fiber extrusion line with a single-screw extruder $(\mathrm{D}=19 \mathrm{~mm}, \mathrm{~L} / \mathrm{D}=25$, Rheomex $\mathrm{OS}$, Thermo Fisher Scientific, Germany) at a processing temperature of $220^{\circ} \mathrm{C}$. A static mixer was installed between extruder and melt pump (Haake OS, Thermo Fisher Scientific) to promote dispersion and distribution of PS. The as-spun monofilament was quenched in a water bath installed beneath the spinneret. The water bath distance is $5 \mathrm{~cm}$ unless mentioned otherwise. The custom-made capillary dies all had a conical entrance zone with a length of $\sim 30 \mathrm{~mm}$ and a cone angle of $2 \theta=26.5^{\circ}$. The morphology development of the polymer blend in capillary die and spinning line were studied by changing capillary length $L_{c a p}$ and capillary diameter $D_{\text {cap }}$ (hence changing the aspect ratio $\left.A R=L_{c a p} / D_{\text {cap }}\right)$, and take-up speed v. $L_{\text {cap }}$ investigated were 23 and $55 \mathrm{~mm}, D_{\text {cap }}$ were 1.0 and $1.5 \mathrm{~mm}$. The take-up speeds $\mathrm{v}$ were $5,10,15,20$ and $30 \mathrm{~m} / \mathrm{min}$. A schematic diagram of the melt extrusion setup can be found in our previous paper ${ }^{[19]}$. Table 1 summarizes the operating parameters of the different extrusion trials performed in the present study. It should 
be noted that, in the case of the low viscosity ratio PP/PS blend filament, the take-up speed was limited to $20 \mathrm{~m} / \mathrm{min}$ due to processing constraints and concerns regarding filament quality.

Table 1. Processing conditions of different melt-spun samples.

\begin{tabular}{|c|c|c|c|c|c|c|c|c|c|c|c|}
\hline $\begin{array}{c}\text { Viscosity } \\
\text { ratio }\end{array}$ & $\begin{array}{c}\text { Capillary } \\
\text { length } \\
{[\mathrm{mm}]}\end{array}$ & $\begin{array}{c}\text { Capillary } \\
\text { AR }\end{array}$ & $\begin{array}{c}\text { Water } \\
\text { bath } \\
\text { distance } \\
{[\mathrm{cm}]}\end{array}$ & $\begin{array}{c}\text { Mass flow } \\
\text { rate } \\
\text { [g/min }]\end{array}$ & $\begin{array}{l}\text { Die exit } \\
\text { velocity } \\
{[\mathrm{m} / \mathrm{min}]}\end{array}$ & $\begin{array}{c}\text { Take-up } \\
\text { speed } \\
{[\mathrm{m} / \mathrm{min}]}\end{array}$ & $\begin{array}{l}\text { Draw- } \\
\text { down } \\
\text { ratio } \\
\text { DDR } \\
\end{array}$ & $\begin{array}{c}\text { Melt } \\
\text { temperature } \\
\text { in die }\left[{ }^{\circ} \mathrm{C}\right]\end{array}$ & $\begin{array}{c}\text { Die } \\
\text { pressure } \\
\text { [bar] }\end{array}$ & $\begin{array}{c}\text { Fiber } \\
\text { diameter } \\
{[\mu \mathrm{m}]}\end{array}$ & $\begin{array}{l}\text { Sample } \\
\text { number }\end{array}$ \\
\hline 2.5 & 35 & 35 & 5 & 4.70 & 6.58 & 10 & 1.5 & 230 & 88 & 762 & 6173 \\
\hline 2.5 & 35 & 35 & 5 & 4.70 & 6.58 & 20 & 3.0 & 230 & 88 & 521 & 6172 \\
\hline 2.5 & 35 & 35 & 5 & 4.70 & 6.58 & 30 & 4.5 & 230 & 88 & 444 & 6174 \\
\hline 2.5 & 35 & 35 & 10 & 4.70 & 6.58 & 20 & 3.0 & 230 & 88 & 545 & 6171 \\
\hline 2.5 & 35 & 35 & 15 & 4.70 & 6.58 & 20 & 3.0 & 230 & 88 & 519 & 6170 \\
\hline 2.5 & 35 & 35 & 20 & 4.70 & 6.58 & 20 & 3.0 & 230 & 88 & 525 & 6169 \\
\hline 2.5 & 55 & 55 & 5 & 3.90 & 5.46 & 10 & 1.8 & 230 & 130 & 708 & 6179 \\
\hline 2.5 & 55 & 55 & 5 & 3.90 & 5.46 & 20 & 3.6 & 230 & 130 & 481 & 6178 \\
\hline 2.5 & 55 & 55 & 5 & 3.90 & 5.46 & 30 & 4.8 & 230 & 130 & 382 & 6180 \\
\hline 2.5 & 55 & 55 & 10 & 3.90 & 5.46 & 20 & 3.6 & 230 & 130 & 475 & 6177 \\
\hline 2.5 & 55 & 55 & 15 & 3.90 & 5.46 & 20 & 3.6 & 230 & 130 & 485 & 6176 \\
\hline 2.5 & 55 & 55 & 20 & 3.90 & 5.46 & 20 & 3.6 & 230 & 130 & 475 & 6175 \\
\hline 0.35 & 55 & 37 & 5 & 4.72 & 3.23 & 5 & 1.5 & 230 & 106 & 998 & 6314 \\
\hline 0.35 & 55 & 37 & 5 & 4.72 & 3.23 & 10 & 3.0 & 230 & 106 & 582 & 6313 \\
\hline 0.35 & 55 & 37 & 5 & 4.72 & 3.23 & 15 & 4.5 & 230 & 106 & 703 & 6312 \\
\hline 0.35 & 55 & 37 & 10 & 4.72 & 3.23 & 20 & 3.0 & 230 & 106 & 490 & 6311 \\
\hline 0.35 & 55 & 37 & 15 & 4.72 & 3.23 & 20 & 3.0 & 230 & 106 & 553 & 6310 \\
\hline 0.35 & 55 & 37 & 20 & 4.72 & 3.23 & 20 & 3.0 & 230 & 106 & 553 & 6309 \\
\hline 0.35 & 35 & 23 & 5 & 5.20 & 2.93 & 5 & 1.7 & 230 & 64 & 1533 & 6321 \\
\hline 0.35 & 35 & 23 & 5 & 5.20 & 2.93 & 10 & 3.4 & 230 & 64 & 1028 & 6320 \\
\hline 0.35 & 35 & 23 & 5 & 5.20 & 2.93 & 20 & 5.1 & 230 & 64 & 757 & 6319 \\
\hline 0.35 & 35 & 23 & 10 & 5.20 & 2.93 & 20 & 3.4 & 230 & 64 & 722 & 6318 \\
\hline 0.35 & 35 & 23 & 15 & 5.20 & 2.93 & 20 & 3.4 & 230 & 64 & 618 & 6317 \\
\hline 0.35 & 35 & 23 & 20 & 5.20 & 2.93 & 20 & 3.4 & 230 & 66 & 710 & 6316 \\
\hline 0.35 & 55 & 55 & 5 & 2.5 & 3.50 & 5 & 1.4 & 230 & 192 & 698 & 6327 \\
\hline 0.35 & 55 & 55 & 5 & 2.5 & 3.50 & 10 & 2.8 & 230 & 192 & 526 & 6326 \\
\hline 0.35 & 55 & 55 & 5 & 2.5 & 3.50 & 20 & 4.2 & 230 & 192 & 357 & 6325 \\
\hline 0.35 & 55 & 55 & 10 & 2.5 & 3.50 & 20 & 2.8 & 230 & 192 & 361 & 6324 \\
\hline 0.35 & 55 & 55 & 15 & 2.5 & 3.50 & 20 & 2.8 & 230 & 192 & 365 & 6323 \\
\hline 0.35 & 55 & 55 & 20 & 2.5 & 3.50 & 20 & 2.8 & 230 & 192 & 366 & 6322 \\
\hline
\end{tabular}

\subsection{Morphology Characterization}

In order to study the morphology of blends, PP/PS blend fibers were carefully fixed in a holder with defined orientation, embedded in epoxy resin, and solidified at room temperature. The embedded samples were polished in a grinder polisher (EcoMet 250 Pro, Buehler, Illinois, USA) to obtain cross and longitudinal sections of fibers. To dissolve the dispersed PS phase at the surface, the polished samples were immersed in tetrahydrofuran (Analytical standard, Aldrich) under ultrasonic conditions for $10 \mathrm{~min}$, and then in ethanol under ultrasonic conditions 
for another $10 \mathrm{~min}$. This process was repeated three times at room temperature to wash away the dissolved parts.

Scanning electron microscopy (SEM, S4800, Hitachi, Japan) was used to scan the section surface across longitudinal and cross fiber sections. For high viscosity ratio system, the entire diagonal of a cross section image was divided into 6 parts and analyzed using the method described in our previous paper ${ }^{[19]}$. In the case of low viscosity ratio PP/PS blend fibers, the radii of PS dispersed phases are too small to analyze with the same method used for high viscosity ratio system. The SEM image of a fiber section from low viscosity ratio PP/PS blend was taken at the corresponding representative position as shown in Figure 1, in order to study the radial morphology difference.

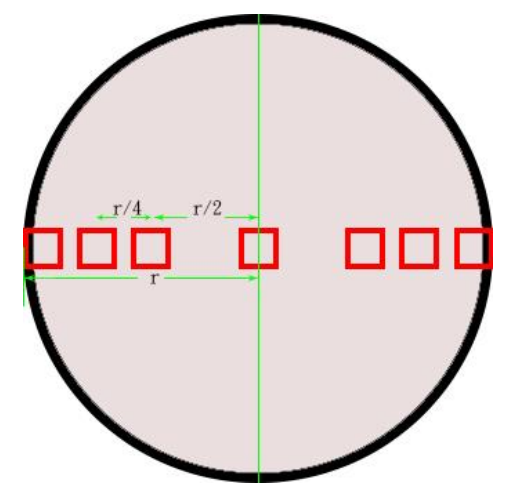

Figure 1. Partitioning of micrographs of a fiber cross section for image analysis.

\subsection{Image Analysis}

Micrographs of the fiber sections were evaluated using image analysis functionalities in MATLAB ${ }^{[40]}$. At least three images for one individual partition were analyzed to achieve appropriate statistics for the low viscosity ratio system. For high viscosity ratio system with sparse distribution of dispersed phases, at least five images for one individual partition were analyzed to achieve appropriate statistics. Typical morphology representations of fiber sections were processed by image analysis. Here, droplet sizes were enlarged 250 times and colored according to a defined scale bar. The droplets were plotted at their corresponding locations in the fiber section. 
In the image analyses, the radius of PS phase is defined as equivalent area radius

$$
\mathrm{r}=\sqrt{\frac{\mathrm{A}}{\pi}}
$$

where $\mathrm{A}$ is the area of the dispersed phase. The number probability $\mathrm{p}_{\mathrm{n}}$ of dispersed phases in different radius ranges is defined as the ratio of the number of droplets in a defined range to the total number of droplets.

$$
\mathrm{p}_{\mathrm{n}}=\frac{\sum \mathrm{n}_{\mathrm{k}<i<\mathrm{j}}}{\sum \mathrm{n}_{\mathrm{i}}}
$$

where $n_{i}$ is the number of dispersed phases with a radius of $r_{i}, n_{k<i<j}$ is the number of dispersed phases within the radius range of $\mathrm{k}-\mathrm{j}$. The average radius of PS phases is defined as

$$
\overline{\mathrm{r}}=\frac{\sum \mathrm{n}_{\mathrm{i}} \mathrm{r}_{\mathrm{i}}}{\sum \mathrm{n}_{\mathrm{i}}}
$$

where $n_{\mathrm{i}}$ is the number of dispersed phases with a radius of $r_{i}$.

\section{Results and Discussions}

Two sets of polymer blends with the same chemistry but different viscosities are studied to illuminate the effect of the viscosity ratio on the emerging gradient morphologies. Figure 2 shows viscosities of PS and PPs, as well as viscosity ratios of PP/PS blends, versus shear rate at $230{ }^{\circ} \mathrm{C}$. High and low viscosity PPs were blended with PS to produce two viscosity ratios of $0.3 \sim 0.8$ and $2 \sim 3$ in the shear rate range studied. The zero shear viscosity $\left(\eta_{0}\right)$ for the polymers were obtained through curve fitting using the three-parameter Bird-Carreau model ${ }^{[41]}$.

$$
\eta=\frac{\eta_{0}}{\left(1+(\lambda \dot{\gamma})^{2}\right)^{\frac{1-n}{2}}}
$$

where $\eta$ is the shear viscosity, $\dot{\gamma}$ the shear rate, $\mathrm{n}$ the power law index, and $\lambda$ the relaxation time. The zero-shear viscosity of low viscosity PP (PP1), high viscosity PP (PP2) and PS are estimated 
to be $\sim 655, \sim 4682$ and $\sim 1617 \mathrm{~Pa} \cdot \mathrm{s}$, respectively. The other fitting parameters of Equation ( 4 ) are given in Table S1 of the Supporting Information. It should be noted that both blends have almost similar elasticity ratios during the processing as shown in Figure S1.
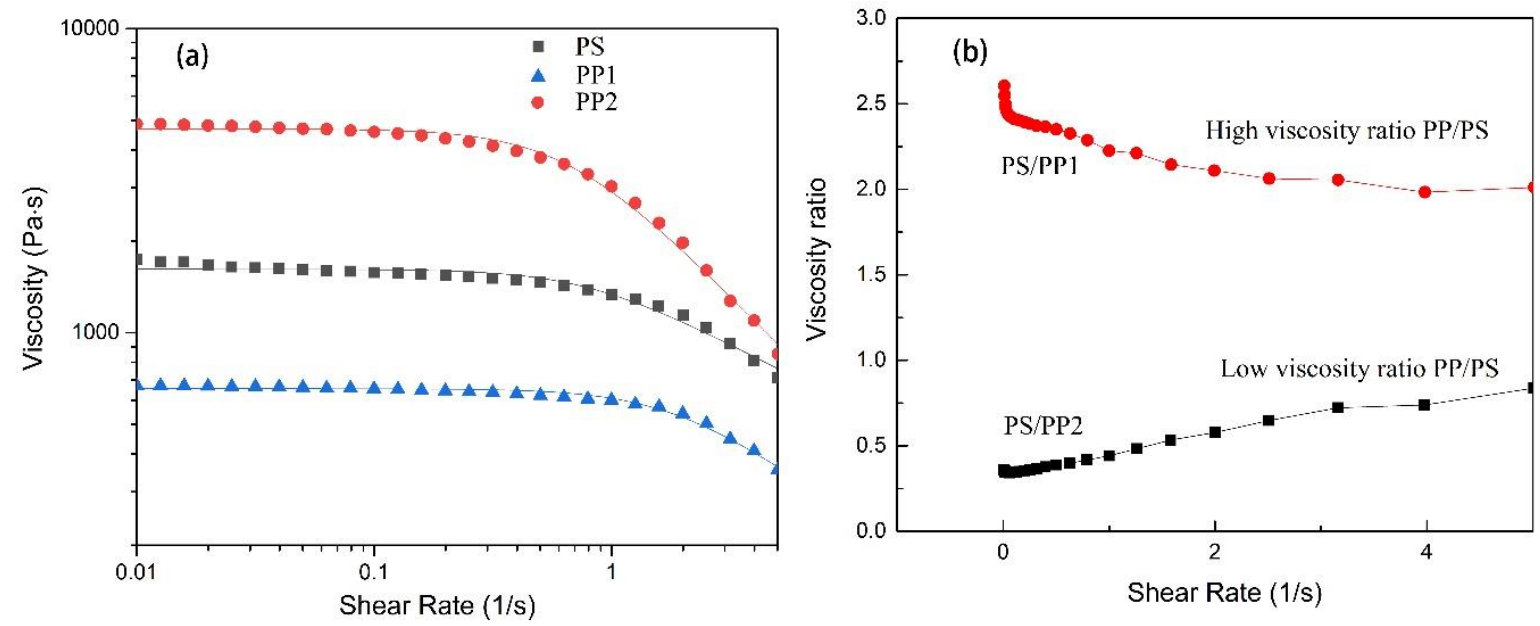

Figure 2. The shear viscosities of PP and PS (a), and the viscosity ratio of PS to PP (b) as a function of shear rate.

Figure 3 shows the typical morphology of fiber cross-sections resulting from melt extrusion trials with different viscosity ratios and capillary ARs. An analysis of the morphology images of cross-sections of PP/PS fibers is displayed in Figure S2 of Supporting Information. The corresponding radial distribution of average radii of PS domains are shown in Figure 4. For all studied cases of high viscosity ratio PP/PS blend fibers, the average radii of PS domains in the center region are larger than those in the surface region. Higher AR strengthens the gradient structure of PP/PS blend fibers, which is in agreement with our previous findings ${ }^{[19]}$. The morphology of low viscosity ratio PP/PS blend fibers, on the other hand, shows an inverse gradient microstructure with small diameter of PS phases in the center region and large irregular PS domains near the surface region. Some irregular domains are also observed in the high viscosity ratio samples, however, they are not as dominant as in the low viscosity ratio samples. Obviously, the viscosity ratio can be utilized as an effective parameter to control the gradient morphology in a given capillary die setup. It is interesting to note that the radial distribution of average radii of PS domains display a W-like profile for the low viscosity ratios. Once the PS 
domains are shaped under the effect of converging flow in the entrance zone and flow into the capillary, PS domains in different radial positions of the die will experience different shear rates. In general, the low viscous PS phase migrates to the die wall where the shear rate is highest ${ }^{[33]}$. These PS domains near the surface region will subsequently coalesce into larger domains and form irregular shapes. The PS domains near the capillary center with weak shear rates merely experience weak deformations. In between these two extremes, a competition between breakup and coalescence forms the W-profile in the cross sections. 

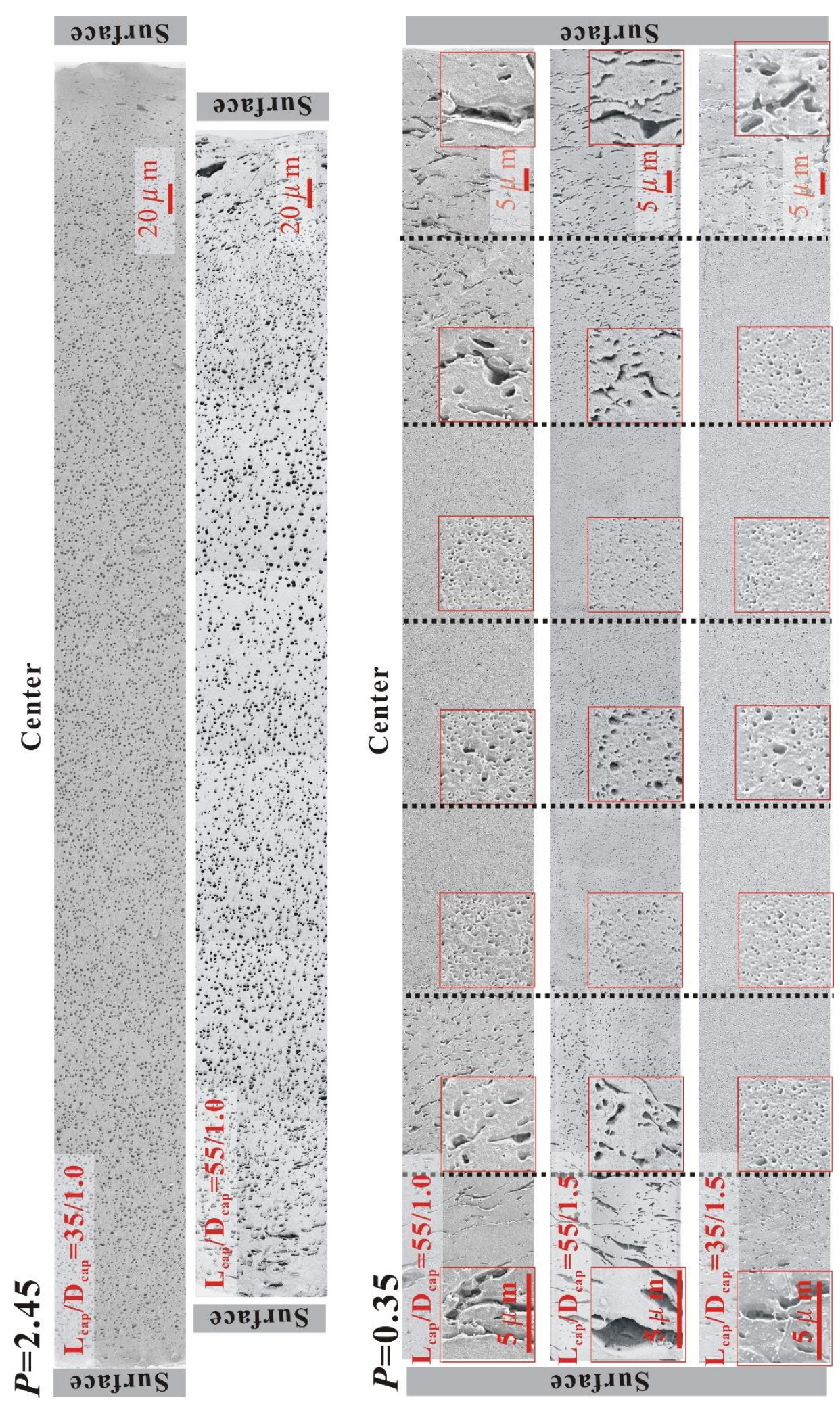

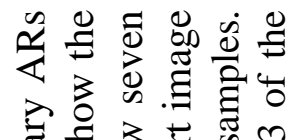

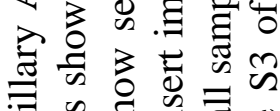

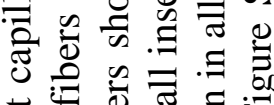

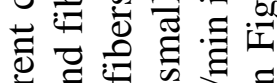
d d क

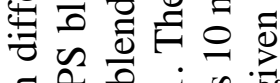

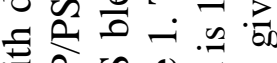

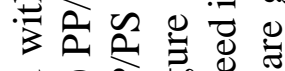

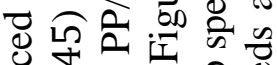

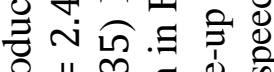
II है 离.

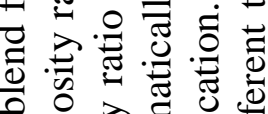

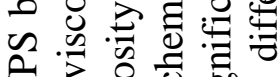

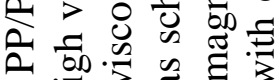
4 긍

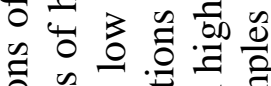

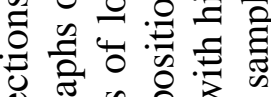

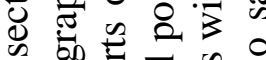

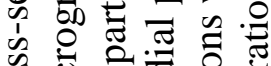

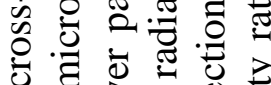

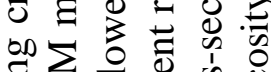

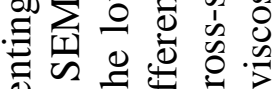
ब

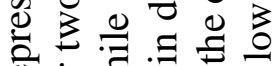
这

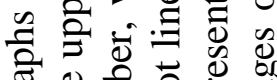

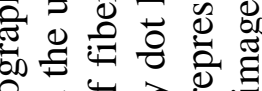
능

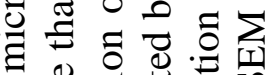

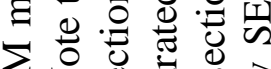

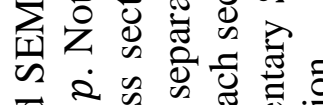

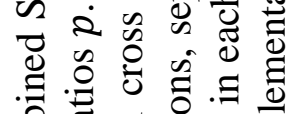
言 专 రิ

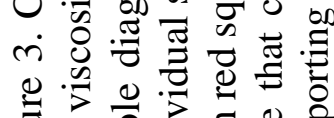

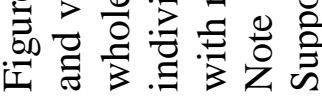



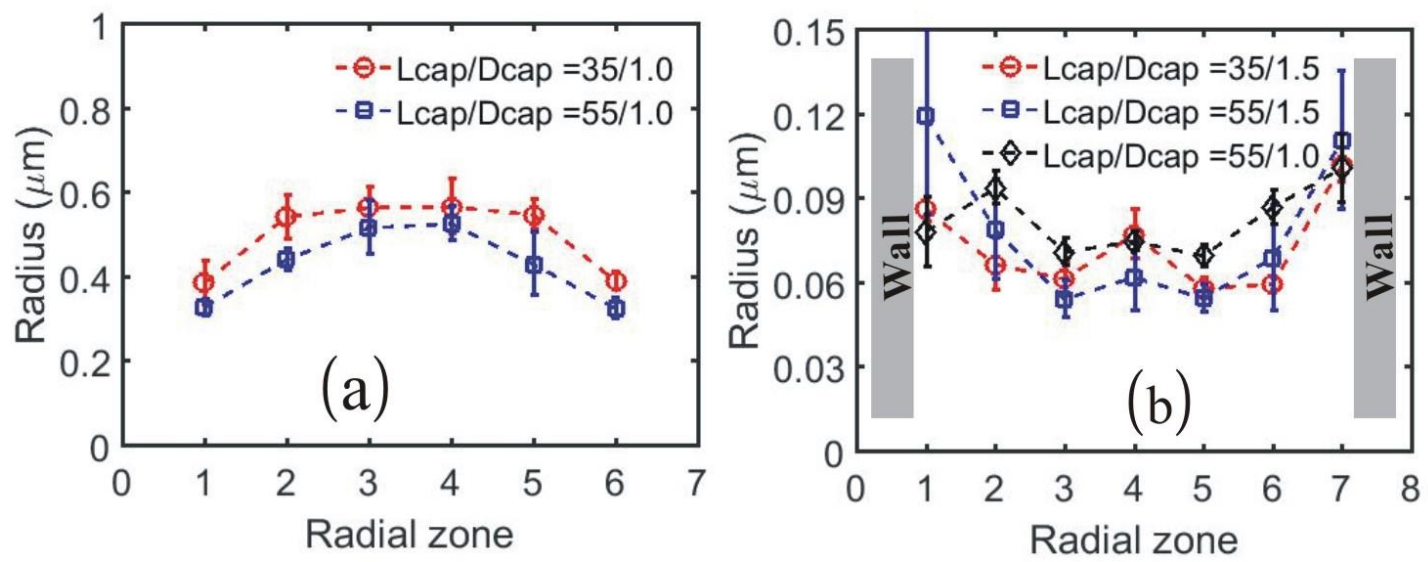

Figure 4. Radial distribution of average radii of PS domains in the cross-sections of different viscosity ratio PP/PS blend fibers melt-spun through capillary dies, (a) $p=2.45$, and (b) $p=0.35$. The take-up speed is $10 \mathrm{~m} / \mathrm{min}$ in all samples. The water bath distance is $5 \mathrm{~cm}$. Note that complementary measurements with different take-up speeds are given in Figure S4 of the Supporting Information.

The occurrence frequencies in terms of the number probability parameter defined in Equation ( 2 ) of large and small droplets dispersed across the fiber cross-section were further investigated, see Figure 5. In blends with low viscosity ratio, the droplets in the fiber center are relatively larger than those in its neighboring sections. The number probability of large droplets decreases with the capillary AR, which is in agreement with our previous investigations ${ }^{[19]}$. The cross-section morphologies merely represent an incomplete picture of the gradient morphology if one does not consider the longitudinal microstructures. Figure 6 shows the combined SEM micrographs of longitudinal sections of PP/PS blend fibers. PS domains in the high viscosity ratio blends appear as fibrils with small diameters near the surface region and as short fibrils with large diameters near the center region. In the low viscosity ratio blends, PS domains form large irregular domains near the surface region and long thin fibrils near the center region. 

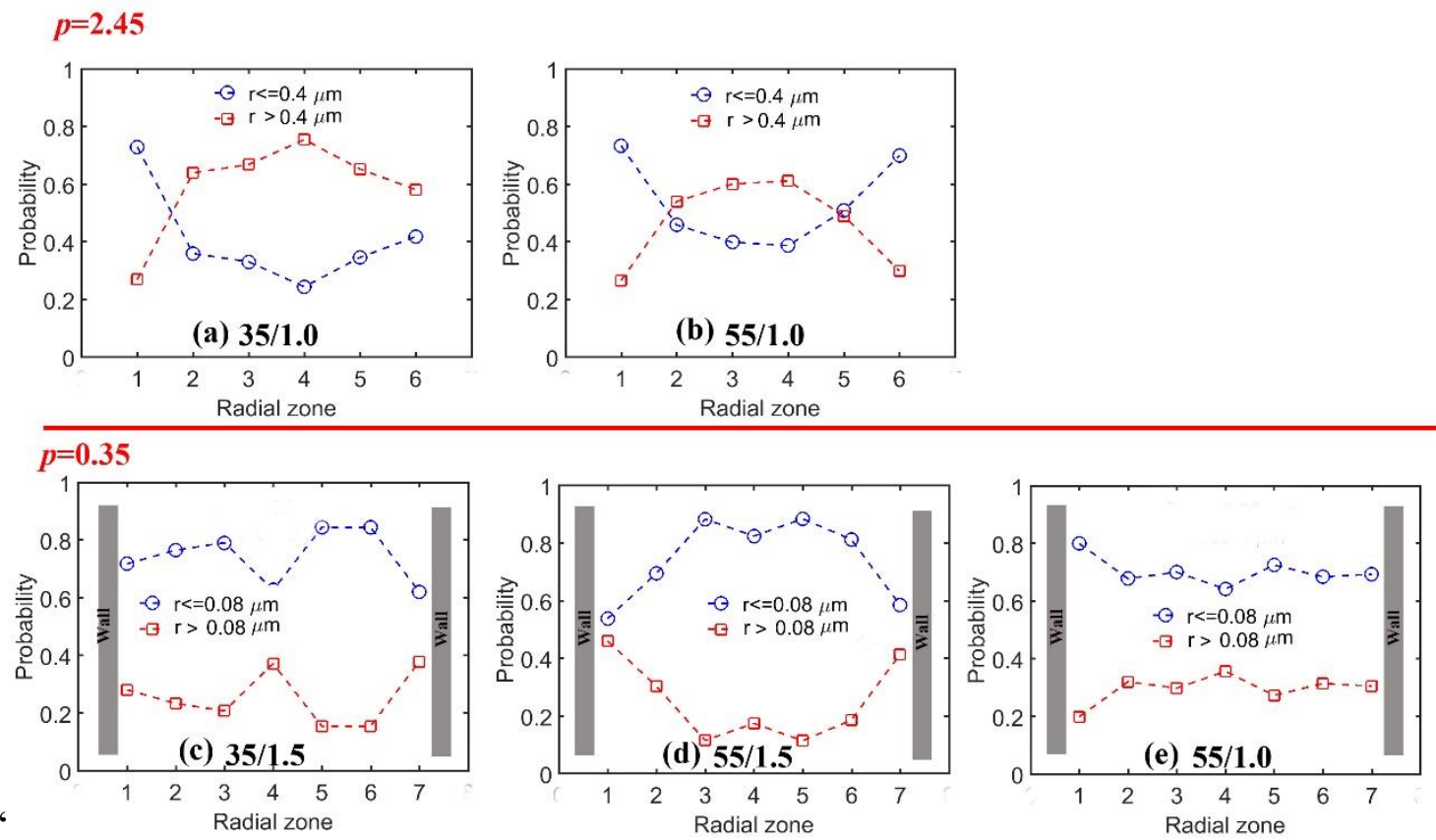

Figure 5. Number probability distribution of PS domains in the fiber cross-section, with different radius ranges of $\mathrm{r} \leq 0.4 \mu \mathrm{m}$ (blue) and $\mathrm{r}>0.4 \mu \mathrm{m}$ (red) in the upper row for high viscosity ratio PP/PS blend fibers produced with capillary (a) $L_{\text {cap }} / D_{\text {cap }}=35 / 1.0$, (b) $L_{\text {cap }} / D_{\text {cap }}=55 / 1.0$, and different radius ranges of $\mathrm{r} \leq 0.08 \mu \mathrm{m}$ (blue) and $\mathrm{r}>0.08 \mu \mathrm{m}$ (red) in lower row for low viscosity ratio PP/PS blend fibers produced with capillary (c) $L_{\text {cap }} / D_{\text {cap }}=35 / 1.5$, (d) $L_{\text {cap }} / D_{\text {cap }}=55 / 1.5$, (e) $L_{\text {cap }} / D_{\text {cap }}=55 / 1.0$. Note that $0.4 \mu \mathrm{m}$ and $0.08 \mu \mathrm{m}$ is a good approximation of the mean droplet radius measured in the central zone of high and low viscosity ratio PP/PS blend fibers (Figure 4), respectively. The take-up speed is $10 \mathrm{~m} / \mathrm{min}$ in all samples. Note that complementary measurements with different take-up speeds are given in Figure S5 of the Supporting Information. 


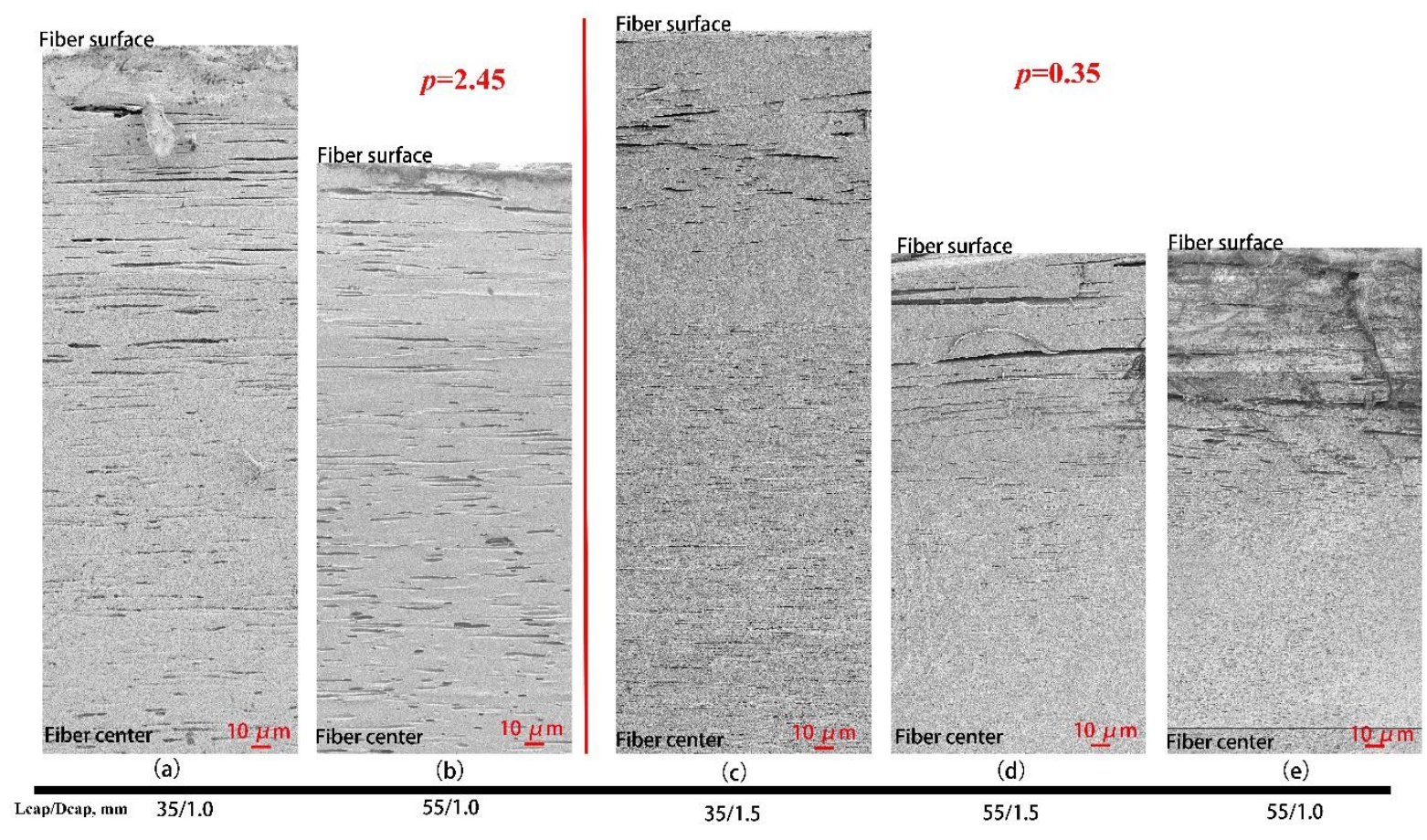

Figure 6. SEM micrographs of (half) longitudinal sections of PP/PS blend fibers produced with different capillary ARs and viscosity ratios. The left two columns show the sections of high viscosity ratio PP/PS blend fibers produced with capillary (a) $L_{c a p} / D_{\text {cap }}=35 / 1.0$, (b) $L_{\text {cap }} / D_{\text {cap }}=55 / 1.0$, the right three columns show the sections of low viscosity ratio PP/PS blend fibers produced with capillary (c) $L_{c a p} / D_{c a p}=35 / 1.5$, (d) $L_{c a p} / D_{c a p}=55 / 1.5$, (e) $L_{c a p} / D_{c a p}=55 / 1.0$. The take-up speed is $10 \mathrm{~m} / \mathrm{min}$ in all samples. Note that complementary SEM images of samples with different take-up speeds are given in the Supporting Information.

According to Taylor's theory ${ }^{[42,43]}$, the deformation and breakup of dispersed droplets can be evaluated by capillary number $\mathrm{Ca}$, which measures the ability to deform or break up droplets under the mechanical equilibrium between viscous forces and interfacial tension in flow. Taylor's original theory is based on the deformation of a single droplet and thus ignores the effects observed in systems with multiple droplets. For such systems, Grace's study ${ }^{[26]}$ shows that the minimum critical capillary number $\mathrm{Ca}_{\mathrm{c}}$ (calculated from $\left.\log \mathrm{Ca}_{\mathrm{c}}=-0.506-0.0994 \log \mathrm{p}+0.124 \log ^{2}(\mathrm{p})-\frac{0.155}{\log -0.611}\right)$ for continuous stretching and breaking up of dispersed phase occurs in the viscosity ratio range between 0.1 and 1 . In this range, the dispersed phase with low viscosity can be deformed into small and uniform dispersed droplets, since the high viscosity matrix can stretch and break the dispersed phase ${ }^{[27,29]}$. This is in agreement with our results, see Figure 3 and Figure 4. The average radius of PS phase in the 
low viscosity ratio PP/PS blend fibers is one order of magnitude smaller than that in the high viscosity ratio system.

During the flow of PP/PS melt in a capillary, the polymer chains experience both elongational and shear stresses. Figure 7 (a) schematically shows the capillary die and the elongational stress profile along the flow axis in the entrance region ${ }^{[39]}$. PS droplets will deform into fibrils in the entrance zone, under the effect of raising elongational stress. Subsequently, the deformed PS phases will pass through the capillary die, where retraction, deformation, breakup and coalescence of dispersed domains can happen when their respective criteria are satisfied. After the PP/PS blend melt exists the capillary, the droplet-matrix morphology is mainly controlled by the take-up action. However, the gradient morphologies are mainly formed before the takeup zone as shown earlier ${ }^{[19]}$. Furthermore, the distance between the spinneret and the water bath $\mathrm{d}$ does not influence the gradient morphology development systematically within the limits and conditions studied here (see Figures S6-S14 in Supporting Information). Therefore, the viscosity ratio as well as the die geometry are the main parameters controlling the gradient microstructures. 


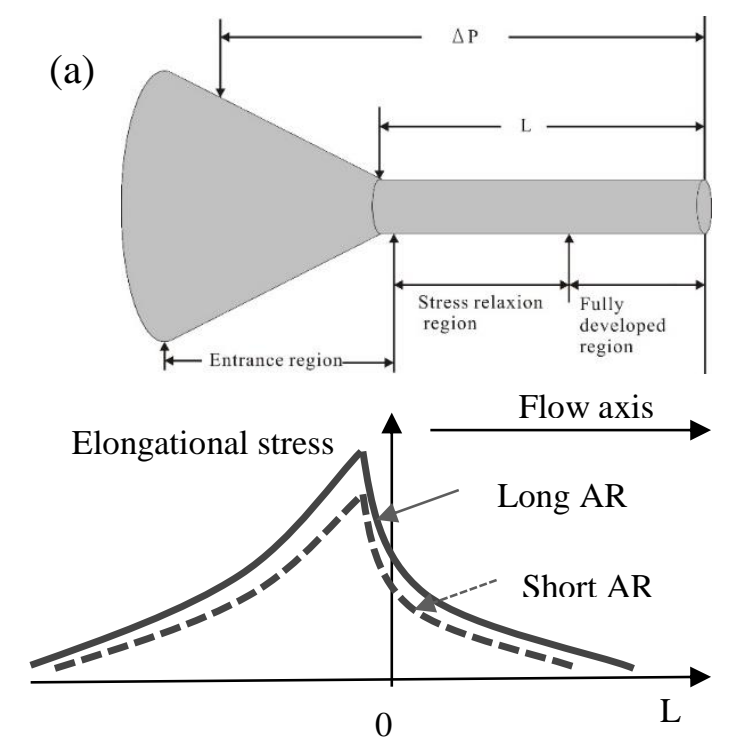

(b)

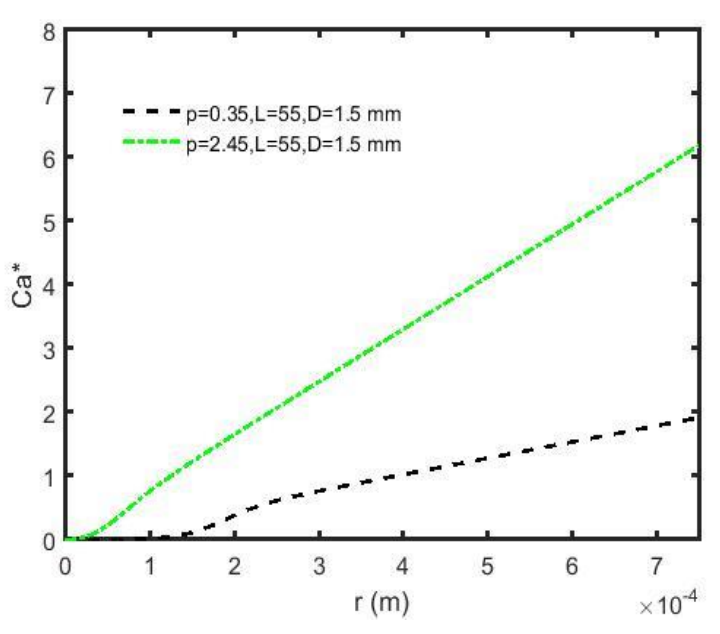

Figure 7. (a) Schematic diagram of capillary die. The upper part schematically describes the working cell diagram including the entrance region, stress relaxion region and fully developed region. The lower part describes the elongational stress distribution along the flow direction in capillary ${ }^{[38,39]}$. (b) Reduced capillary number $\mathrm{Ca}^{*}$ of PS droplets in different viscous PP. The shear rate profile is calculated from velocity gradient. The velocity profile is calculated from the parabolic equation $V(r)=\frac{Q}{\pi R^{2}}\left(\frac{3 n+1}{n+1}\right)\left(1-\left(\frac{r}{R}\right)^{\frac{n+1}{n}}\right)$. The viscosity is determined by using Bird-Carreau model (equation ( 4 )) at calculated shear rates. Interfacial tension is calculated from Palmer's equation ${ }^{[44]}$ to be $4.64 \mathrm{mN} / \mathrm{m}$. $\mathrm{R}_{0}$ for high and low viscosity ratio systems is estimated $\sim 0.4$ and $0.08 \mu \mathrm{m}$, respectively, from the average volume of the central zone in Figure 6.

A theoretical understanding of the morphology evolution can be provided in terms of the reduced capillary number $\mathrm{Ca}^{*}=\mathrm{Ca} / \mathrm{Ca}_{\mathrm{C}}{ }^{[16,42]}$. Figure 7 (b) shows $\mathrm{Ca}^{*}$ in different viscosity ratio systems processed in the same capillary die. In the low viscosity ratio system, the $\mathrm{Ca}^{*}$ of PS phases in the center region $(r=0-0.1 \mathrm{~mm})$ is practically 0 . Hence, the viscous force is not strong enough to deform droplets, and already deformed droplets will retract after the entrance region. This explains the occurrence of larger droplets near the center line of the capillary in low viscosity ratio system. In this system, $\mathrm{Ca}^{*}$ is between 0.1 and 1 near the capillary center $(\mathrm{r}=0.1-0.4 \mathrm{~mm})$, which indicates that PS droplets deform with minor breakups during flow in this region. In regions close to the capillary wall, $\mathrm{Ca}^{*}$ is in the range of $1-4$, hence, PS droplets still deform but also have a higher probability to break up in this region ${ }^{[42]}$. The increasing $\mathrm{Ca}^{*}$ from the centerline to the wall increases the probability of droplets to deform into fibrils and 
break up near the wall. Moreover, the phase with lower viscosity can also migrate to the surface of the polymer blend during melt processing and undergo coalescence processes due to the higher shear rates ${ }^{[33]}$. As for the blend with a high viscosity ratio, the $\mathrm{Ca}^{*}$ is larger than 1 in most radial positions of the capillary and reaches values larger than 4 near the surface region. This means that the PS domains continually deform into fibrils with low probability for droplet breakups. Consequently, in the low viscosity ratio systems, the coalescence of continuously deforming PS droplets plays a key role in the formation of irregular shapes particularly observed closer to the walls. Figure 8 shows the surface and cross-section morphology of a low viscosity ratio PP/PS blend fiber near the surface. As can be seen, coarse patterns originating from large irregular PS domains are observed close to the fiber surface. To design such structures, further information is required on a nanoscopic level regarding the molecular behaviors at the surface of the fibers interacting with the die walls ${ }^{[45]}$.

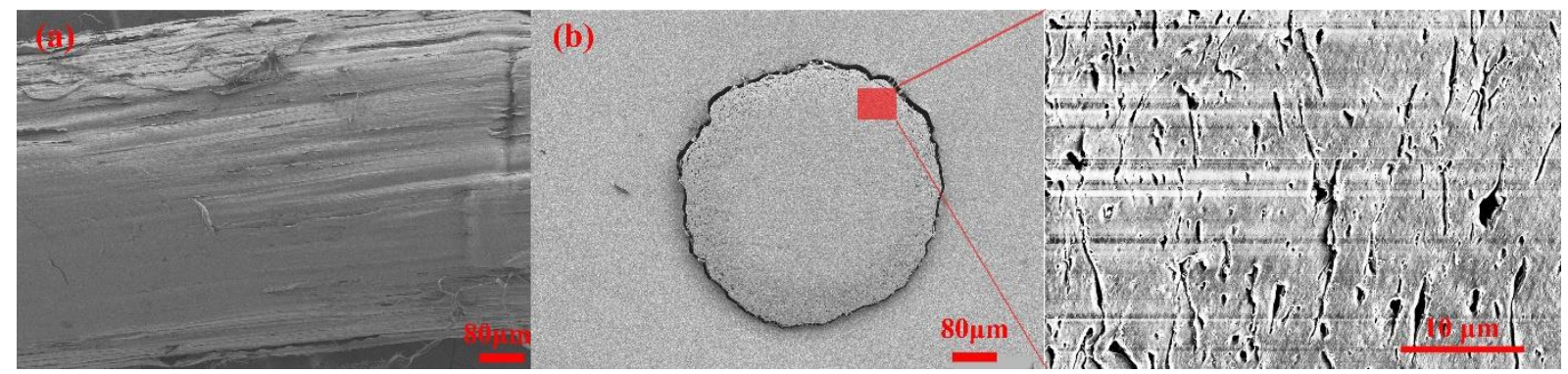

Figure 8. SEM micrographs of a PP/PS blend fiber with low viscosity ratio: (a) fiber surface after dissolving with THF, (b) cross section after dissolving with THF.

According to the results presented so far, the viscosity ratio is an effective parameter to control the gradient morphology. The features of such gradient PP/PS fibers can be inspiring for the design of melt-spun functional materials. For instance, the large domains of second phase in the surface region could serve as a carrier of functional additives for fibers and composites. The second phase close to the surface can also alter the surface properties without changing the properties of matrix polymer. The transparent second phases with cross-sectional sizes comparable to wavelengths of visible and near-infrared light could act as scattering centers and enhance the light reflectance of the fibers ${ }^{[46]}$. The filamentary phases enable the fiber to guide 
light along their longitudinal direction through transverse Anderson localization, which could find potential application in optical signal and image transport ${ }^{[46,47]}$.

\section{Conclusions}

Gradient PP/PS blend fibers were prepared by melt-spinning with distinct inverse gradient microstructures in bulk. The dispersed phase in the high viscosity ratio system showed long thin fibrils in the surface region and large deformed droplet near the center region. On the contrary, the PS domains in the low viscosity ratio system form irregular domains near the surface region, and long thin fibrils near the center region. These two opposite gradient microstructures result from the parabolic shear rate profile in the capillary die due to differences in hydrodynamics of PS domains in low viscosity and high viscosity PP matrix. These features are mainly controlled by the viscosity ratio as well as the die geometry. Thus, the viscosity ratio can act as a powerful tool to manipulate the gradient morphology in a given processing setup. In light of the presented results, further research must be carried out to reveal the role of viscosity ratio against other material properties such as elasticity ratio, interfacial tension, and blend composition.

\section{References}

[1] C. R. A. Catlow. The structural science of functional materials [J]. IUCrJ. 2018, 5(Pt 1): 1-3.

[2] Ali Gooneie, Stephan Schuschnigg, Clemens Holzer. A Review of Multiscale Computational Methods in Polymeric Materials [J]. Polymers. 2017, 9(12): 16.

[3] Ali Gooneie, Pietro Simonetti, Khalifah A. Salmeia, Sabyasachi Gaan, Rudolf Hufenus, Manfred P. Heuberger. Enhanced PET processing with organophosphorus additive: Flame retardant products with added-value for recycling [J]. Polymer Degradation and Stability. 2019, 160: 218-228.

[4] Y. Liang, L. Li, R. A. Scott, K. L. Kiick. Polymeric Biomaterials: Diverse Functions Enabled by Advances in Macromolecular Chemistry [J]. Macromolecules. 2017, 50(2): 483-502.

[5] George Polymeropoulos, George Zapsas, Konstantinos Ntetsikas, Panayiotis Bilalis, Yves Gnanou, Nikos Hadjichristidis. 50th Anniversary Perspective: Polymers with Complex Architectures [J]. Macromolecules. 2017, 50(4): 1253-1290.

[6] Yurong Yan, Ali Gooneie, Haixian Ye, Lingli Deng, Zhiming Qiu, Felix A. Reifler, Rudolf Hufenus. Morphology and Crystallization of Biobased Polyamide 56 Blended with Polyethylene Terephthalate [J]. Macromolecular Materials and Engineering. 2018, 303(9): 1800214.

[7] Khalifah A. Salmeia, Ali Gooneie, Pietro Simonetti, Rashid Nazir, Jean-Pierre Kaiser, Alexandra Rippl, Cordula Hirsch, Sandro Lehner, Patrick Rupper, Rudolf Hufenus, Sabyasachi Gaan. Comprehensive 
study on flame retardant polyesters from phosphorus additives [J]. Polymer Degradation and Stability. 2018, 155: 22-34.

[8] María P. Fernández-Ronco, Boguslawa Gradzik, Ali Gooneie, Rudolf Hufenus, Miroslawa El Fray. Tuning Poly(3-hydroxybutyrate) (P3HB) Properties by Tailored Segmented Biocopolymers [J]. ACS Sustainable Chemistry \& Engineering. 2017, 5(11): 11060-11068.

[9] Ali Gooneie, Stephan Schuschnigg, Clemens Holzer. Dissipative Particle Dynamics Models of Orientation of Weakly-Interacting Anisometric Silicate Particles in Polymer Melts under Shear Flow: Comparison with the Standard Orientation Models [J]. Macromolecular Theory \& Simulations. 2016, 25(3): 287-302.

[10] Lyudmila M. Bronstein, Anna Ivanovskaya, Tom Mates, Niels Holten-Andersen, Galen D. Stucky. Bioinspired Gradient Materials via Blending of Polymer Electrolytes and Applying Electric Forces [J]. The Journal of Physical Chemistry B. 2009, 113(3): 647-655.

[11] C. F. Jasso-Gastinel. Gradients in Homopolymers, Blends, and Copolymers [M]. in Modification of Polymer Properties, C.F. Jasso-Gastinel and J.M. Kenny, Editors. William Andrew Publishing, 2017: 185-210.

[12] S. R. Djafari Petroudy. 3 - Physical and mechanical properties of natural fibers [M]. in Advanced High Strength Natural Fibre Composites in Construction, M. Fan and F. Fu, Editors. Woodhead Publishing, 2017: 59-83.

[13] S. Amada, Y. Ichikawa, T. Munekata, Y. Nagase, H. Shimizu. Fiber texture and mechanical graded structure of bamboo [J]. Composites Part B-Engineering. 1997, 28(1-2): 13-20.

[14] Xu-Ming Xie, Yue Chen, Zeng-Min Zhang, Akihiko Tanioka, Masami Matsuoka, Kenji Takemura. Controls of Gradient Morphology and Surface Properties of Polymer Blends [J]. Macromolecules. 1999, 32(13): 4424-4429.

[15] C. E. Rogers. Transport through polymer membranes with a gradient of inhomogeneity [J]. Journal of Polymer Science Part C: Polymer Symposia. 1965, 10(1): 93-102.

[16] Qiang Xing, Meifang Zhu, Yiheng Wang, Yanmo Chen, Yu Zhang, J. Pionteck, H. J. Adler. In situ gradient nano-scale fibril formation during polypropylene (PP)/polystyrene (PS) composite fine fiber processing [J]. Polymer. 2005, 46(14): 5406-5416.

[17] Houkang He, Long Chen, Shanshan Sun, Tonghui Wang, Yu Zhang, Meifang Zhu. Study on the matrixfibril morphologies of polypropylene/polystyrene blends under non-isothermal uniaxial elongational flow [J]. Fibers and Polymers. 2014, 15(4): 744-752.

[18] Dan Pan, Long Chen, Houkang He, Kangwei Deng, Zongyi Qin. Deformation of dispersed polystyrene droplets in immiscible polypropylene/polystyrene blend fibers under uniaxial elongational flow [J]. Fibers and Polymers. 2016, 17(9): 1343-1351.

[19] Dan Pan, Rudolf Hufenus, Zongyi Qin, Long Chen, Ali Gooneie. Tailored Gradient Morphologies and Anisotropic Surface Patterns in Polymer Blends [J]. Macromolecular Materials and Engineering. 2018, 304(3): 1800601.

[20] B. D. Favis, J. P. Chalifoux. The effect of viscosity ratio on the morphology of polypropylene/polycarbonate blends during processing [J]. Polymer Engineering \& Science. 1987, 27(21): 1591-1600.

[21] Marco Dressler, Brian J. Edwards, Erich J. Windhab. An examination of droplet deformation and break-up between concentrically rotating cylinders [J]. Journal of Non-Newtonian Fluid Mechanics. 2008, 152(13): 86-100.

[22] Massimiliano Gabriele, Rossana Pasquino, Nino Grizzuti. Effects of Viscosity-Controlled Interfacial Mobility on the Coalescence of Immiscible Polymer Blends [J]. Macromolecular Materials and Engineering. 2011, 296(3-4): 263-269.

[23] A. Gooneie, H. Nazockdast, F. Shahsavan. Effect of selective localization of carbon nanotubes in pa6 dispersed phase of PP/PA6 blends on the morphology evolution with time, part 2: Relaxation of deformed droplets after cessation of flow [J]. Polymer Engineering \& Science. 2016, 56(1): 51-60.

[24] G. I. Taylor. The Formation of Emulsions in Definable Fields of Flow [J]. Proceedings of the Royal Society A: Mathematical, Physical and Engineering Sciences. 1934, 146(858): 501-523.

[25] Cox R G. The deformation of a drop in a general time-dependent fluid flow [J]. Journal of Fluid Mechanics. 1969, 37(3): 601-623.

[26] Grace H P. Dispersion phenomena in high viscosity immiscible fluid systems and application of static mixers as dispersion devices in such systems [J]. Chemical Engineering Communications. 1982, 14: 225 277. 
[27] Xiang Lu, Jianqing Zhao, Xiaoyun Yang, Peng Xiao. Morphology and properties of biodegradable poly (lactic acid)/poly (butylene adipate-co-terephthalate) blends with different viscosity ratio [J]. Polymer Testing. 2017, 60: 58-67.

[28] J. Cailloux, T. Abt, V. Garcia-Masabet, O. Santana, M. Sanchez-Soto, F. Carrasco, M. Ll Maspoch. Effect of the viscosity ratio on the PLA/PA10.10 bioblends morphology and mechanical properties [J]. Express Polymer Letters. 2018, 12(6): 569-582.

[29] Long Wang, Lian-Fang Feng, Xue-Ping Gu, Cai-Liang Zhang. Influences of the Viscosity Ratio and Processing Conditions on the Formation of Highly Oriented Ribbons in Polymer Blends by Tape Extrusion [J]. Industrial \& Engineering Chemistry Research. 2015, 54(44): 11080-11086.

[30] Xin Yi, Ling Xu, Yu-Ling Wang, Gan-Ji Zhong, Xu Ji, Zhong-Ming Li. Morphology and properties of isotactic polypropylene/poly(ethylene terephthalate) in situ microfibrillar reinforced blends: Influence of viscosity ratio [J]. European Polymer Journal. 2010, 46(4): 719-730.

[31] R. N. Jones, K. Walters. Coextrusion of polymer melts [M]. in Rheological fundamentals of Polymer Processing, J.A. Covas, et al., Editors. Netherlands: Springer, 1995: 265-288.

[32] Kaijian Wu, Jinchao Yu, Jiaqing Yang, Shenghui Chen, Xiaofeng Wang, Yumei Zhang, Huaping Wang. Properties and phase morphology of cellulose/aromatic polysulfonamide alloy fibers regulated by the viscosity ratio of solution [J]. Cellulose. 2018, 25(2): 903-914.

[33] Esma Ayad, Aurélie Cayla, François Rault, Anne Gonthier, Christine Campagne, Eric Devaux. Effect of Viscosity Ratio of Two Immiscible Polymers on Morphology in Bicomponent Melt Spinning Fibers [J]. Advances in Polymer Technology. 2018, 37(4): 1134-1141.

[34] Stefano Guido. Shear-induced droplet deformation: Effects of confined geometry and viscoelasticity [J]. Current Opinion in Colloid \& Interface Science. 2011, 16(1): 61-70.

[35] James L. White, Kyonsuku Min. The development of phase morphology and polymer chain orientation in the processing of incompatible polymer blends [J]. Advances in Polymer Technology. 1985, 5(4): 225237.

[36] S. Barwinkel, A. Seidel, S. Hobeika, R. Hufen, M. Morl, V. Altstadt. Morphology Formation in PC/ABS Blends during Thermal Processing and the Effect of the Viscosity Ratio of Blend Partners [J]. Materials (Basel). 2016, 9(8): 659-678.

[37] Yong-Juan Gao, Zheng-Ying Liu, Chao-Lu Yin, Shi-Lin Huang, Ming-Bo Yang. Preparing iPP/HDPE/CB functionally gradient materials: influence factors of components and processing [J]. Polymers for Advanced Technologies. 2012, 23(3): 695-701.

[38] James H. Han, Chin Choi-Feng, De-Jie Li, Chang Dae Han. Effect of flow geometry on the rheology of dispersed two-phase blends of polystyrene and poly(methyl methacrylate) [J]. Polymer. 1995, 36(12): 2451-2462.

[39] V. I. Brizitsky, G. V. Vinogradov, A. I. Isayev, Y. Y. Podolsky. Extensional stresses during polymer flow in ducts [J]. Journal of Applied Polymer Science. 1978, 22(3): 751-767.

[40] MATLAB. Http://Www.Mathworks.Com/. 2017 [cited 2018 July 20].

[41] Tim Osswald, Natalie Rudolph. Polymer Rheology [M]. in Polymer RheologyCarl Hanser Verlag GmbH \& Co. KG, 2014: I-XI.

[42] M.A. Huneault, Z.H. Shi, L.A. Utracki. Development of polymer blend morphology during compounding in a twin-screw extruder. Part IV A new computational model with coalescence [J]. Polymer Engineering \& Science. 1995, 35(1): 115-127.

[43] G. I. Taylor. The Viscosity of a Fluid Containing Small Drops of Another Fluid [J]. Proceedings of the Royal Society A: Mathematical, Physical and Engineering Sciences. 1932, 138(834): 41-48.

[44] Guillermo Palmer, Nicole R. Demarquette. Evaluation of imbedded fiber retraction phenomenological models for determining interfacial tension between molten polymers [J]. Polymer. 2005, 46(19): 81698177.

[45] Ali Gooneie, Joamin Gonzalez-Gutierrez, Clemens Holzer. Atomistic Modelling of Confined Polypropylene Chains between Ferric Oxide Substrates at Melt Temperature [J]. Polymers. 2016, 8(10): 361.

[46] N. N. Shi, C. C. Tsai, M. J. Carter, J. Mandal, A. C. Overvig, M. Y. Sfeir, M. Lu, C. L. Craig, G. D. Bernard, Y. Yang, N. Yu. Nanostructured fibers as a versatile photonic platform: radiative cooling and waveguiding through transverse Anderson localization [J]. Light Sci Appl. 2018, 7: 37-46. 
[47] S. Karbasi, R. J. Frazier, K. W. Koch, T. Hawkins, J. Ballato, A. Mafi. Image transport through a disordered optical fibre mediated by transverse Anderson localization [J]. Nat Commun. 2014, 5: 3362-3371. 